\title{
The Art of Global Information Civilization
}

\author{
System Analysis
}

\author{
Oleg Pobol \\ Razumovsky Moscow State University of Technology and Management \\ Moscow, Russia \\ e-mail: pobolo@rambler.ru
}

\begin{abstract}
This article focuses on the analysis of art in the post-industrial society of the information civilization, in the context of the general systems theory, the demonstration of the properties of the art as a variety of a techno-genic system belonging to automated technosphere, and the determination of the development prospects within the noospheric civilization.
\end{abstract}

Keywords-culture; art; information; technosphere; noosphere; synergetics; system; model

\section{INTRODUCTION}

By the close of the 20th century the development of the industrial society saw the emergence of a global postindustrial information civilization. The previous mechanistic scientific paradigm, implying antecedence of the material substance, has been succeeded by the information paradigm based on the prime significance of information in reference to its material implementation. Alongside with that, a new comprehensive worldview of the information civilization is evolving.

According to the scientific in-system worldview, any object or process is examined during the research and management as a system: it is a cyclic generality made up of subordinate subsystems and yet it constitutes an element of a higher hierarchy system. Here it is customary to consider the original theory as the generalized common information space, the particular instance of which is all objects and fields known today. The Universe appears as an information dynamic super-system. The matter of various nuances of the same wave nature presents the realization of the macrocosm information space - its logosphere.

The Universe is defined by an across-the-board integration of the objects-subsystems; each making up a component of the whole with the proper development cycle, yet exhibiting properties of synergetic self-organization, i.e. rationality of the corresponding hierarchic level. Each object of our world is composed of substance matter of different fineness levels, as if shells of inertial real kernel, serving for the receive-transmit of energy information and being in continuous interaction with the environment.
Life and reason in the cycling movement are the natural and eternal forms of existence of matter and information. Information is the generative frame of the Universe. Its synergetic model is an informational super-system made up of subsystems of the living intelligent Universe, united around the same general goal or idea. Global awareness is the fundamental factor of reality, while the human consciousness constitutes one of its parts. However, the human being appears to not be the reasonable Supreme Being.

The world is considered interconnected and interdependent in both place and time. We do not see this relationship in complex systems, but we are aware of them. They are provided with one wave nature of the information and substance, and with the relevant single information code - "In the beginning was the Word." Man existing in the biosphere environment of infoenergy and substance matter (IES) is a functional element of the Universe - a global constituent, built within its informational field.

Culture of the socium is literally: worship of the beam of truth, a tool of learning and portraying the ambience properties (including the man) and the body of knowledge at the same time. Conceptually, the culture preserved by traditions as an IES matrix is maintaining the logos communication with Earth and the man who is evolving while getting to know the Universe. The culture models the socium and manages its development, designating the management criteria. Depending on the instruments of knowledge, the culture breaks down into science, art, religion and everyday culture. The science, which discerns not between good and bad, is the principal form of cognition in the contemporary culture. Here the truth is the best solution to the original problem. The art ad verbum is the study of a subject in itself. This is a specific method of the sensory and intuitive cognition of the reality and relationship between its constituents, intended to produce esthetically expressive forms and models of reality.

The process of scientific knowledge obtainment, extenuated by intelligent machines, ended up in the late 20th century by globalization, entailing formation of an artificial environment: the global technosphere, comprised of automated man-machine pools, the technogenic systems (TS), and the formation of a single information management 
architecture. The art in this constitutes one of the technosphere subsystems, a particular TS form.

Owing to the special importance of the art's informational impact on vital processes of the civilization, it is important to analyze its properties from the point of view of the General Systems theory. The present research is developing and concretizing the generalities of the study [1].

\section{INFORMATION MODEL OF THE ART}

Addressing this research task in hand requires looking into the fundamentals of the system analysis and Informatiology [2-5] applicable to the range of issues under consideration and singling out the information systems' peculiar properties in the context of the art.

The System is defined as the integrity, composed of elements that are united by the shared objective, the universal interaction algorithm and the functional environment of exchanging data, energy and substance. The information presents the knowledge about changes in the world and processes occurring in it (in this case, it is the system of art forms and its symbols) possessing fundamental properties: novelty, relevance, validity, objectivity, integrity, value, and aesthetic expressiveness.

Interaction with an object-system and its study results in the development of a model - a simplified substituting image, a system prototype (a visualization, an image, a product, a description, a sound patter, a formula, etc.). This image is never equivalent to the object, but establishes connection with it and is used for particular problem solutions. The information model of the object (here we talk about the model of subsystems and infoenergy communication channels in the form of visual and sound symbols and related thought forms) describes the importance for this review parameters and variables of the object. By providing the model with data on changes in the input information it permits the modeling of an object's possible conditions. The generic data model as applied to Earth as the controlled object, considering understanding of the Informatiology and the systems theory, is given in the Fig.1. Inside the infosphere of the Universum's logosphere there is a formed project/model named "Earth" with own information field (1), its perceptible physical achievement in the process of evolution, the macrocosm, (2) and the invisible microcosm/nanoworld (3). It is evident that all vital processes in the super-system Universum are supplied with the corresponding infoenergy and material substance flows using the single information code.

While the general laws of nature have been specified by the Universal information field (the Universum logosphere), the program variants of the world of ideas on Earth are determined by the infosphere of the environment 1 , and implemented in space and time in the world of subjects 2 out of the microcosm substance 3 . The process of the humankind's creativity awareness is going in the sphere 1-2.

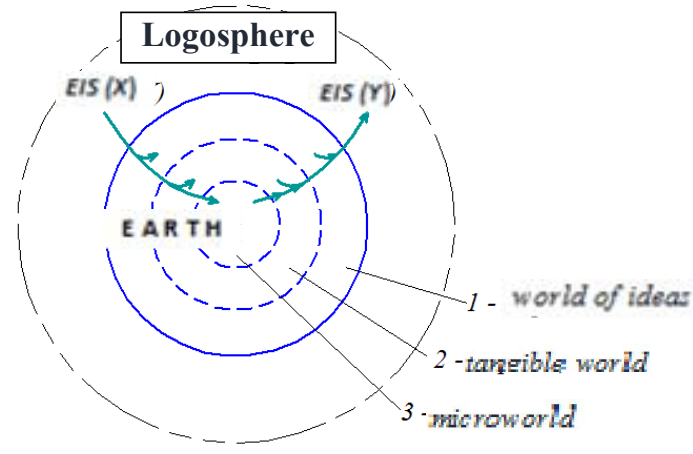

Fig.1. General information model of the system "Earth"

The artificial environment of modern humankind, the technosphere, is composed of industrial and nonindustrial TS - automated man-machine systems, while the art makes one of such systems. The TS are synergetic systems, whose general properties manifest in a self-organized complex hierarchical structure, arranged from the highest to lowest. The purpose of the technosphere, for which it has been initially created, is the biosphere processing into products of consumption, the market economy goods. This target has been set by the liberalism of the information civilization's consumer society.

While deciding practical problems it is customary to present the complicated systems as a total of simpler element subsystems, in other words, to apply the reduction method. The thus found model properties of the subsystem elements are carried over to an object of higher hierarchy, as the traditional approach demands, ignoring its system attributes. Furthermore, it is usually overlooked that the reviewed performance of an object belonging to a lowerhierarchy-level modeling differs fundamentally from its integral model performance in the highest-level hierarchies.

Recognition of an object's system attributes allows us to understand its performance from a new angle and to obtain conceptually new results. Each system appears in such a case as a subsystem of a higher level, and its specific nature is defined by integral properties essential for the performance of a higher hierarchic level system. System attributes are determined primarily by its structure, namely, by the pattern of relationship between its elements that ensures energy-mass and data exchange between the subsystems, rather than by its subsystems' characteristics. The object under research is singled out as an entity with properties, which are exposed when it comes into play as one of the components of a higher hierarchic level; the components of the proper object are singled out subsequently. Such three-tier representation of the compound object is the most effective.

For an object of art, as of a technogenic system, we may single out in a generalized sense four life phases that pertain to the conceptualization and design; to the production of an object of art; to the scope of its operation and consumption; and to its recycling. Referring to Fig.2, the input of the infoenergy and material substance EIS(X) transforms over 
the course of the TS activity into objects of the art (i.e. reality patterns) in the shape of the EIS(Y) at the output. Creative specialists assisted by automated machines handle the process throughout all its stages. Moreover, the magnitude of information technologies is constantly increasing in the course of the technosphere evolution: Intelligent machines replace the creative personality, which is not surprising inasmuch as they were initially designed to replace the man and convert the biosphere into products of consumption.

Each TS, as any system, has a single control center with its hierarchic structure. Concerning the infosphere the theory system distinguishes a three-level control structure with the higher conceptual (strategic), middle adaptation (tactic), and lower (operational) levels (Fig.2).

The design and administration of the synergetic models, which constitute the general (global) project, are achieved at the tactic level (2). The coordination/operational level (1) is where the administration of stand-alone particular creative projects within complex synergetic models is executed.

Globalization delivers the process of humankind and automated technosphere management from the single center in compliance with the ideology of the socium. This ideology presents a conceptual system of political, legal, ethical, esthetical, religious, and philosophical commitments - through which reality is realized and assessed.

The liberal ideology of the modern global information civilization, due to passivity of social consciousness, rests on the scientific materialist paradigm of the industrial civilization. Selection of managers for all levels of administration is carried out in line with the operational ideology, and the higher the level, the more severe the conformity.

Operators involved in the system life processes usually apprehend only the lower, coordination and operational levels of management, while all principal issues and problems turn up and are resolved at the higher levels of tactical and strategic management. Decisions here are made in compliance with the ideological affiliation of the socium. Benefit makes the key criterion of the decision quality assessment. These issues were fully considered in the work [1].

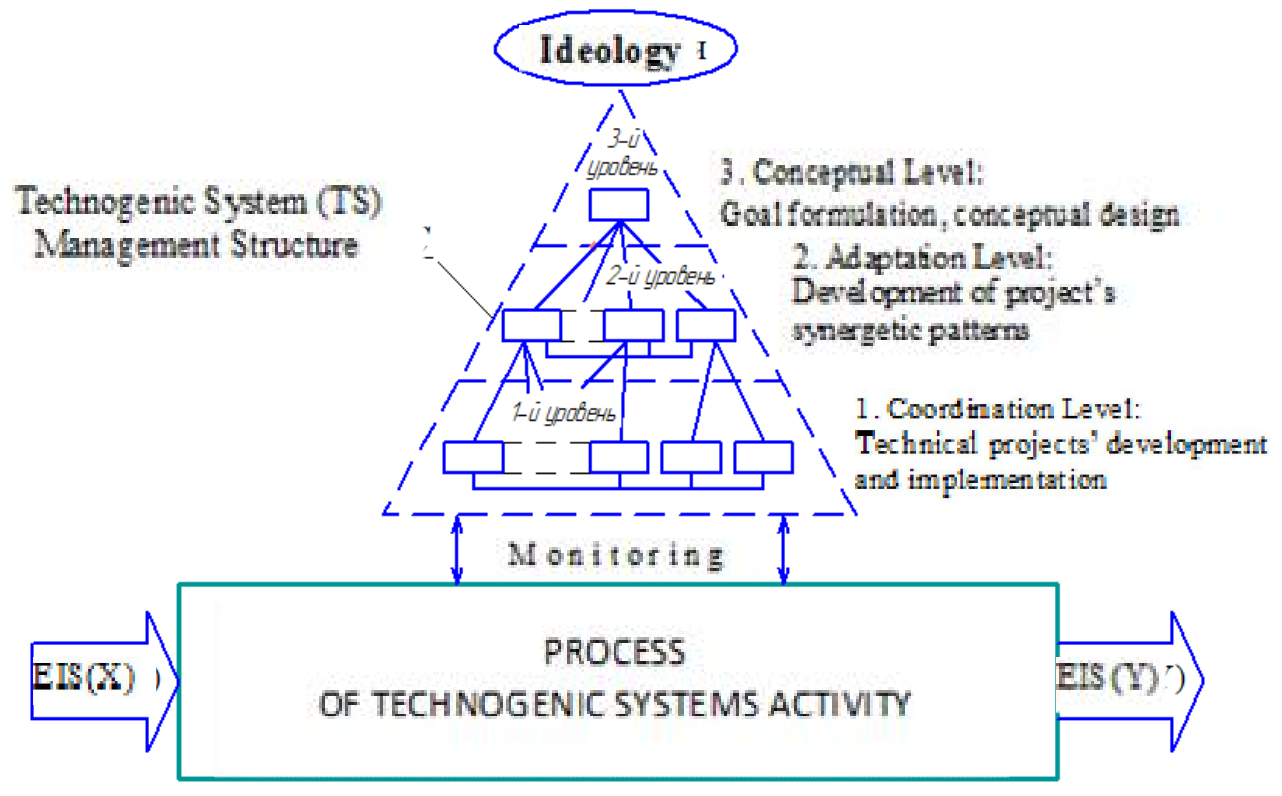

Fig.2. Information model of TS "Art" 
According to the TS information model "Art", the creative process of development and implementation of a particular project can be presented as a graphic pattern, shown in Fig.3.

The automated decision support system's capability of a purposeful creative behavior provides its memory with the thesaurus (Tes) - an information pattern of the environment and itself. The system builds the thesaurus knowledge during the course of specialist training, with the addition of specialized knowledge pools and libraries and interaction with the Earth's infosphere and technosphere during its vital processes. The TS is able to control the behavior (to the extent of the socium ideology, hierarchy and specialists' skills) depending on the specified target package $Z$. In addition, the system is geared to a particular task, set by a structure of a higher-level hierarchy, and concurrently factors in the common target assignments of Earth's infosphere and the technosphere of the socium. At the output of such a goal-oriented system with the operator R, the performance results $\mathrm{Y}$ vary depending on the external action $\mathrm{X}$ and self-target assessment $\mathrm{X} *(\mathrm{t}, \mathrm{y})$ upon the expected result $\mathrm{Y}^{*}$ in current time, when the goal is attained. The form of the system operator $\mathrm{R}$ corresponds the form of the art, so that one and the same real-world object is rendered through various artistic images and means. In all instances, however, this image is shaped by a technogenic system relevant to its properties and particulars of understanding the truth, and with the commitment of the higher-level control structure. The technosphere, as shown in Fig.2 and Fig.3, blocks the EIS-stream of Earth's infosphere.

According to the above considered information patterns, the creative process is executed and developed in the EISmatrix of postindustrial multi-culture. Here, just as in the social consciousness, prevalent is the ideology of materialist paradigm of the matter primacy that is underlying the scientific worldview of the industrial civilization, modern education and upbringing system. The liberal ideology of the socium is constraining its development and the development of culture in positive direction vectored by the information paradigm.

\section{PROPERTIES OF SYNERGETIC SYSTEMS}

Aware of its purpose, and endowed with reason and remembering, the system is developing in accordance with a certain project in the information field that interacts with other systems' information fields and is inlined into virtual projects of systems belonging to higher hierarchies of the technosphere, into culture and into the project of Earth's information field.

The self-developing synergetic systems' general characteristic is the presence of a fixed and static time internal factor of its properties under continually varying technogenic and cultural environment conditions. Their principal properties with due regard to the TS peculiarities, which are determined in the present case by the availability of the intelligent machine factor, have been fully considered in the work [1].

Mentality and Anti-tolerance - the system is intelligent and live, and is aware at its level, namely, in its project informational field, of the objectives and rationale specifically pertaining to expansion of the area of effect through the displacement of objects of traditional cultural environment. The system's primary life processes are realized in the multicultural environment through information technologies.

Hierarchy Principle - each object comprises of an element of a higher-level system that assigns to it a particular target, given data communications with adjacent systems. The target system is determined by the ideological orientation of the global technosphere.

Informativity and Anthropity - each subject, developing particular properties of a higher-level hierarchy, holds latent data about the entire infosphere of Earth. This information encrypted in the universal code can be perceived and depicted through various art forms and visualizations.

Openness - a continuous exchange of IES-flows with the environment as the system is developing, and exposes its properties in the course of reasonable interaction with the cultural environment within its ideology.

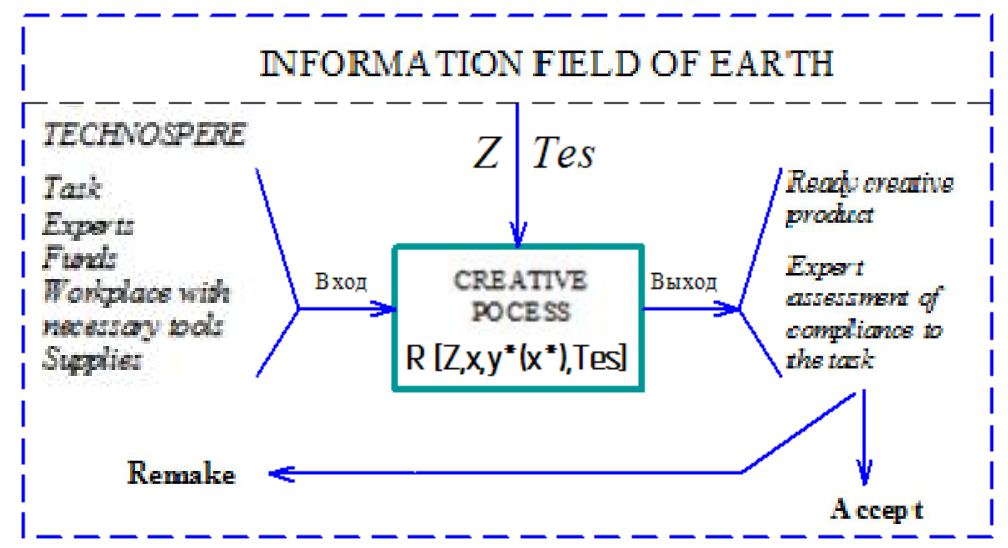

Fig.3. Information model of the creative process 
Structuredness - stability of the network of constituent subsystems' dynamic relationships and the capability to describe the system through the establishment of its structure. The system properties are established most significantly by the communication system between its constituents and the environment. Invisible infoenergy links in time and space, secured through traditions, are of prime importance. The links exhibit dissipativity, i.e. the loss of their content and filling with wrong informational content, which is typical to artwork when traditions are lost.

Integrity - conceptual irreducibility of the system properties to a sum of qualities of the constituent subsystems. A newly formed unity always exceeds the totality of its constituents, which is an especially important factor in the estimation of the creativity syncretic forms' capabilities (architecture, design, television, cinema and theater). The integrity operates in emergence, i.e. in the synergetic system's intrinsic principal properties, missing in the constituent subsystems.

Corporativity - under extreme bifurcation conditions, which is typical to the stance of modern civilization, an element recognizes the entire system and coordinates its behavior both with adjacent and remote elements of diverse hierarchies.

Cyclicity and Dimensionality - each object is moving and developing in harmonic rhythms of its own, and in higher levels that establish inter-relations in the space-andtime process of the system's activity (for instance, cultural environment). It makes itself felt in the continuous change of styles and forms, and is especially accelerated in the transition zones.

Polarity - the simultaneity of the process of development and degradation, action and reaction, intelligibility in direct and back reading. Degradation signifies the beginning of ascendancy in new transition.

Nonequilibrium and Nonlinearity - pertaining to all evolving systems, the spontaneous (intuitive) origination of new local formations, emergence of subjects' new properties, phases to self-organization and the fixation of system new properties. In the state of nonequilibrium of the socium, typical to modern times, bifurcational mechanisms of the existing short-term furcation points in passing to one or another relatively long-term system mode - attractor (stable informational state). The nonlinearity is observed in the distinct disproportion between the input and output signals, whereas an infinitely large response may correspond to an infinitely small impact and vice versa. In this context, alteration or deletion of a component dramatically changes the orientation and intention of an object of art.

By generalizing these properties it can be noticed that the art of modern information civilization offers one of the technosphere's technogenic systems, the activity of which at large and of its subsystems in particular should be understood as a realizable conceptual project of globalization. This is the system of planned and controlled processes of attaining set goals - the biosphere processing into products of consumption having particular timeframes, limited resources and finances to achieve the desired results. The heart of the global liberal socium project is the development in the consumer society of a pluralistic multiculturalism, forcing out the traditional art forms. The role of the art here is the production of attractive standardized and unified machine consumption products. Furthermore, the highest achievement, i.e. the truth pursuant to the scientific paradigm, is considered to be the model that guarantees the best task solution through the preset economic evaluation criteria.

The mentality and anti-tolerance properties of the technogenic systems explain the deliberate and expanding impact of modern art in terms of substitution of the traditional cultural forms. The forms of the art alone, its techniques in particular, providing their implementation cyclicity and polarity, bear a clear-cut machine nature, and increasingly apply the computer IT solutions.

The involvement of the man and his role in creating art masterpieces is decreasing under robotization of the production processes. Concurrently with the de-emphasis of the creative personality's role in the technosphere, are increasing the surplus of human resources and their devaluation.

The properties of the TS completeness and synergism that ensure subsystems' combined action in one direction are distinctly pronounced. The technosphere's cumulative effect far exceeds the effect of each and every component, threatening the existence of entire Earth biosphere.

\section{CONCLUSION}

With reference to the system analysis results given above, it is possible to determine the development outlook for the art of the postindustrial civilization. Understandably, the inadmissible catastrophic development outlook for the logosphere of Universum and information space of Earth should be excluded from consideration. Evolution of Humanity implies, given the implementation of nonequilibrium and nonlinearity properties, the fluctuationbifurcational option of the polar cyclic transformation/transfiguration from the information civilization to the noosphere civilization with the alteration of the paradigm $[1,3,6]$.

Modern art should be considered as one form of transition towards a higher level of development within the ecological paradigm of the all-encompassing unity of the world of ideas and the world of objects of the biosphere. Art in the noospheric culture with its harmonizing commitment to the moral beauty of the austerity instead of an unrestrained consumption should take the paramount position in relation to the science. It will require an adequate transformation of the education and upbringing system.

Abandonment of the liberal ideology of individual purposiveness to the noospheric ideology of love and 
ascesis (the ideology of unity of interests) is expected to restore art's commitment to the cognition of the Universum space's supreme reality and to the creation of society's harmonization esthetic forms and habitat. Therefore, based on the heritage, a noospheric civilization's ecological culture is emerging, achieving a conscious coevolution of humankind and the Earth's biosphere.

\section{REFERENCES}

[1] O. Pobol and G. Firsov, "Hierarchy of communications and cultural environment of the global technogenic civilization," Advances in Social Science, Education and Humanities Research, vol. 3. Paris: Atlantis Press, 2014, pp. 734-738.

[2] E. N. Knyazeva and S.P. Kurdyumov, Synergetics Bases: Synergetic Worldview. M.: LIBROKOM Publishing House, 2010.

[3] I .I. Yuzvishin, Principles of Informatiology. M.: Vysshaya Shkola, 2000.

[4] V. N. Volkova and V.N. Kozlov, System analysis and decisionmaking: Glossary. M.: Vysshaya Shkola, 2004.

[5] I. Prigogine and I. Stengers, Order out of chaos. M.: LKI, 2008.

[6] S. Grof, E. Laszlo and P. Russell, The Consciousness Revolution. M.: K. Kravchuk Publishing House, 2004. 